\title{
Efficient reconfigurable scheme for the recovery of sub-Nyquist sampled sparse multi- band signals
}

Anu Kalidas Muralidharan Pillai and Håkan Johansson

\author{
Linköping University Post Print
}

\section{Tweet}

N.B.: When citing this work, cite the original article.

(C2013 IEEE. Personal use of this material is permitted. However, permission to reprint/republish this material for advertising or promotional purposes or for creating new collective works for resale or redistribution to servers or lists, or to reuse any copyrighted component of this work in other works must be obtained from the IEEE.

Anu Kalidas Muralidharan Pillai and Håkan Johansson, Efficient reconfigurable scheme for the recovery of sub-Nyquist sampled sparse multi-band signals, 2013, IEEE Global Conference on Signal and Information Processing (GlobalSIP 2013), December 3-5, 2013, Austin, Texas, USA.

http://dx.doi.org/10.1109/GlobalSIP.2013.6737146

Postprint available at: Linköping University Electronic Press

http://urn.kb.se/resolve?urn=urn:nbn:se:liu:diva-100968 


\title{
Efficient Reconfigurable Scheme for the Recovery of Sub-Nyquist Sampled Sparse Multi-Band Signals
}

\author{
Anu Kalidas Muralidharan Pillai and Håkan Johansson \\ Division of Electronics Systems, Department of Electrical Engineering, Linköping University, SE-581 83, Sweden \\ Email: \{kalidas, hakanj\}@isy.liu.se
}

\begin{abstract}
Sub-Nyquist sampling makes use of sparsities in analog signals to sample them at a rate lower than the Nyquist rate. The reduction in sampling rate, however, comes at the cost of additional digital signal processing (DSP) which is required to reconstruct the uniformly sampled sequence at the output of the sub-Nyquist sampling analog-to-digital converter. At present, this additional processing is computationally intensive and time consuming and offsets the gains obtained from the reduced sampling rate. This paper focuses on sparse multi-band signals where the user band locations can change from time to time and the reconstructor requires real-time redesign. We propose a technique that can reduce the computational complexity of the reconstructor. At the same time, the proposed scheme simplifies the online reconfigurability of the reconstructor.
\end{abstract}

\section{INTRODUCTION}

It is well known that uniform sampling of a signal which is bandlimited to $f<f_{0}$, at a sampling frequency of $f_{s} \geq 2 f_{0}$, results in a uniformly spaced sequence of samples that can be used to reconstruct the original signal. However, in many cases, the signal is spare in a sense that the actual information is contained in a bandwidth much less than $f_{0}$. One example is a frequency-hopping spread-spectrum communication system where there are one or more narrowband carriers (active subbands) that change their center frequencies within the band $\left[0, f_{0}\right)$ at a certain switching rate. In other words, such signals are locally narrowband (in a time frame) but globally wideband (over several time frames). In such cases, the traditional approach would require a high-speed analogto-digital converter (ADC) operating at a rate of $f_{s} \geq 2 f_{0}$. Hence, within a time frame, the signal is heavily oversampled and the ADC will unnecessarily consume a substantial amount of power.

Sub-Nyquist sampling is becoming increasingly popular in wideband communication systems, especially in batterypowered applications where high-speed uniform sampling results in higher power consumption. In such sampling schemes, the average sampling rate can be much lower than $2 f_{0}$ but still large enough to capture the information content in the signal. In this paper, we focus on the multi-band (or multicoset) sampling approach where the use of cyclic nonuniform sampling (CNUS) helps to reduce the average sampling rate to (in principle) the Landau minimal sampling rate which is determined by the frequency occupancy [1]. It is known that, given the sampling pattern for the CNUS approach, the reconstruction can be done via a set of ideal multilevel synthesis filters [2]. However, the straightforward CNUS reconstruction filters have very high design and computational complexity. Also, in spread-spectrum communication systems where the active subband locations are different for different time frames, the reconstruction scheme should support online reconfigurability without increasing the complexity.

In this paper, we propose a new reconstruction scheme that can substantially reduce the computational complexity. It also facilitates a very fast real-time redesign as it requires a change in only a few multiplier coefficients. Following this introduction, in Section II, we look at the basics of subNyquist sampling in the context of sparse multi-band signals. The proposed reconstruction scheme will be introduced in Section III. This will be followed by Section IV where we will illustrate, using a design example, the savings obtained from the proposed scheme in terms of the design and the computational complexity. Section V concludes the paper.

\section{Sub-Nyquist SAMPling OF Sparse Multi-BAnd SIGNALS}

Assume that $x_{a}(t)$ is a real-valued continuous-time signal that carries information within the frequency band $\omega \in$ $\left(-2 \pi f_{0}, 2 \pi f_{0}\right), f_{0}<1 /(2 T)$. Uniform sampling of $x_{a}(t)$ at a sampling frequency of $f_{s}=1 / T$ results in a discrete-time sequence $x(n)=x_{a}(n T)$. For the sake of simplicity, hereafter we assume that $T=1$. Now it is assumed that the band $\omega \in[0, \pi]$ is divided in to $M$ granularity bands $q_{i}, i=0,1, \ldots, M-1$, of equal width $\pi / M$. In sparse multi-band signals, at any given time frame, only $K$ of the $M$ granularity bands $(K<M)$ are allocated to users. A user can occupy one or several consecutive granularity bands. Further, to be able to design practical filters, we assume a certain amount of redundancy (oversampling) which corresponds to transition bands between user bands. In case of such sparse multi-band signals, uniform sampling will generate more samples than what is required to prevent information loss. The number of samples that is generated during the sampling process can be reduced by using CNUS which only uses a subset $x\left(M n-m_{k}\right), k=1,2, \ldots, K$ with $m_{k} \in[0,1, \ldots, M-1]$ of the uniform samples $x(n)$. A practical implementation of the CNUS is an $M$-channel timeinterleaved ADC (TI-ADC) [3] where only a subset of the channels are used. A reconstructor can be used to recover the uniformly sampled sequence $x(n)$ from $x\left(M n-m_{k}\right)$ for a given set of $K$ granularity bands, by properly selecting the sampling instants $m_{k}$ [4]. 


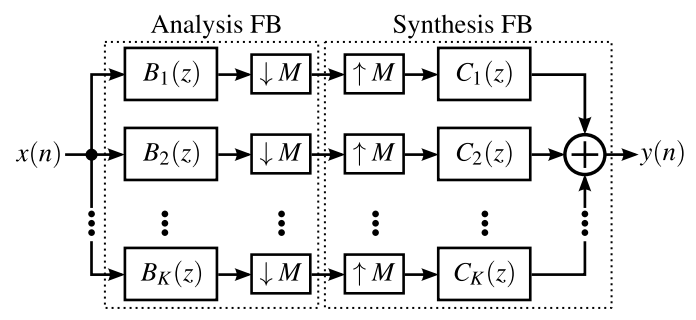

Fig. 1. Reconstruction of sub-Nyquist sampled sparse multi-band signal using analysis and synthesis filters.

It was shown in [2] that perfect reconstruction (PR) can be achieved using ideal multi-level synthesis filters $C_{k}(z)$. This is equivalent to setting the analysis filters $B_{k}(z)$ in Fig. 1 to pure delays $z^{-m_{k}}$. Perfect reconstruction is generally not feasible with realizable filters. However, in practice, it is sufficient to determine $C_{k}(z)$ such that PR is approximated within a given tolerance. This can be done by designing $C_{k}(z)$ straightforwardly, assuming no a priori relations between the filters. However, the reconstructor thus designed may become intolerably costly in real-time application as the computational complexity of this approach is $N K / M$, where $N$ is the filter order of $C_{k}(z)$. Also, at a later time frame, if the location of the $K$ bands change, then all $C_{k}(z)$ need redesign. The design complexity of $C_{k}(z)$ is high as regular filter design is expensive and time consuming. In this paper, to reduce the complexity, we describe the reconstruction in terms of both the analysis and synthesis filters. Unlike in [2] where the analysis filters $B_{k}(z)$ are pure delays, in the proposed method, the filters $B_{k}(z)$ are bandpass decimation filters whose nonzero polyphase components are generalized fractional-delay (FD) filters [5].

\section{Proposed Reconfigurable ReCONStRuCtion SCHEME}

In this section, we propose a reconstruction scheme for sub-Nyquist sampled sparse multi-band signals. The proposed reconstructor can be easily reconfigured if the location of the $K$ active bands change. The point of departure is to describe the reconstruction in terms of both the analysis and synthesis filters as shown in Fig. 1. Each analysis filter $B_{k}(z)$ extracts one of the $K$ active granularity bands. The filtering by $B_{k}(z)$ is followed by downsampling by $M$ so as to have the extracted active granularity band at the lower sampling rate $f_{s} / M$. The low-rate signal is then placed at the original active granularity band location at the higher rate $f_{s}$ via upsampling by $M$ followed by bandpass filtering via $C_{k}(z)$. The synthesis filters $C_{k}(z)$ thus provide a bank of $K$ different bandpass filters.

\section{A. Realization of Analysis Filters}

Recall that, using polyphase decomposition, $B_{k}(z)$ can be represented as [6]

$$
B_{k}(z)=\sum_{m=0}^{M-1} z^{-m} B_{k m}\left(z^{M}\right)
$$

where $B_{k m}(z)$ denotes the polyphase components according to

$$
B_{k m}(z)=\sum_{n=-\infty}^{\infty} b_{k}(n M+m) z^{-n}
$$

with $b_{k}(n)$ denoting the impulse response of $B_{k}(z)$. If the input signal is sub-Nyquist sampled as explained in Section II, the inputs to $M-K$ polyphase branches of $B_{k}(z)$ will be equal to zero. This implies that (1) becomes

$$
B_{k}(z)=\sum_{\ell=1}^{K} z^{-m_{\ell}} B_{k m_{\ell}}\left(z^{M}\right)
$$

where $m_{\ell} \in[0,1, \ldots, M-1], \ell=1,2, \ldots, K$, are the $K$ sampling instants and $B_{k m_{\ell}}\left(e^{j \omega}\right)$ are the $K$ non-zero polyphase components of $B_{k}(z)$. In a full-length paper under way [7], we show that the non-zero polyphase components of the bandpass filter $B_{k}(z)$ are generalized FD filters so that

$$
B_{k m_{\ell}}\left(e^{j \omega}\right) \approx \beta_{k m_{\ell}} e^{j\left(\omega m_{\ell} / M+\alpha_{k m_{\ell}} \operatorname{sgn}(\omega)\right)}, \omega \in[-\pi, \pi] .
$$

In (4), $\beta_{k m_{\ell}}$ and $\alpha_{k m_{\ell}}$ are the modulus and angle, respectively, of a corresponding complex constant $c_{k m_{\ell}}$. It is shown in [7] that the vector $\mathbf{c}_{k}$, containing all the $K$ complex constants $c_{k m_{\ell}}$, $m_{\ell} \in[0,1, \ldots, M-1], \ell=1,2, \ldots, K$, can be determined using matrix inversion as

$$
\mathbf{c}_{k}=\mathbf{A}^{-1} \mathbf{b}_{k}
$$

Here, $\mathbf{A}$ is a $K \times K$ matrix with elements $a_{n_{\ell} m_{\ell}}=e^{j 2 \pi n_{\ell} m_{\ell} / M}$, $n_{\ell}, m_{\ell} \in 0,1, \ldots, M-1, \ell=1,2, \ldots, K$, determined by the $K$ sampling points $m_{\ell}$ and the $K$ active subbands $n_{\ell}$. Further, one of the elements of $\mathbf{b}_{k}$ is unity, its position being determined by the active subband $n_{\ell}$. The remaining elements in $\mathbf{b}_{k}$ are zero.

Since the polyphase components of each $B_{k}(z)$ are as given in (4), all the analysis filters can be expressed with a common set of fixed subfilters, $F_{k}(z)$ and $G_{k}(z)$. The different analysis filters are then obtained via different pairs of values of $\beta_{k m_{\ell}}$ and $\theta_{k m_{\ell}}=\alpha_{k m_{\ell}}+\pi / 4^{1}$ such that

$$
B_{k m_{\ell}}(z)=\beta_{k m_{\ell}}\left[\cos \left(\theta_{k m_{\ell}}\right) F_{k}(z)+\sin \left(\theta_{k m_{\ell}}\right) G_{k}(z)\right]
$$

where

$$
F_{k}\left(e^{j \omega}\right) \approx e^{j \omega m_{k} / M}, G_{k}\left(e^{j \omega}\right) \approx \operatorname{sgn}(\omega) \times j e^{j \omega m_{k} / M} .
$$

\section{B. Realization of Synthesis Filters}

The fixed bandpass synthesis filters $C_{k}(z)$ can be efficiently realized using a cosine modulated FB. A lowpass filter with cutoff frequency at $\pi / 2 M$ is used as the prototype filter [6]. The overall complexity of the synthesis FB correspond to that of the prototype filter plus the cost of a real or complex transform block. By using a fast-transform algorithm, the cost of such a transform block can be made small when compared to the cost of the filters.

\footnotetext{
${ }^{1}$ An additional phase of $\pi / 4$ is required to ensure proper matching between adjacent analysis and synthesis filters in the case of overlapping granularity bands and when cosine modulated FBs are used. This is similar to the additional constants used for matching in conventional cosine modulated FBs [6].
} 


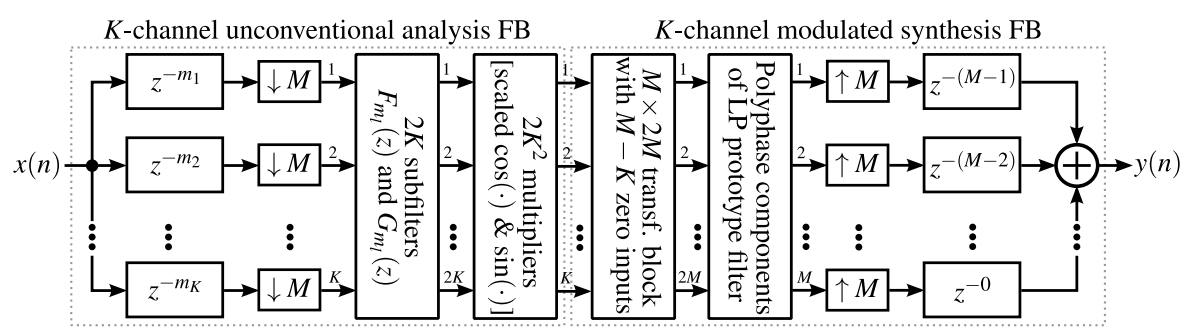

Fig. 2. Proposed reconfigurable reconstruction scheme.

\section{Design of Reconfigurable Reconstructors}

The proposed reconstruction scheme is shown in Fig. 2. The Fourier transform at the output can be written as

$$
Y\left(e^{j \omega}\right)=V_{0}\left(e^{j \omega}\right) X\left(e^{j \omega}\right)+\sum_{m=1}^{M-1} V_{m}\left(e^{j \omega}\right) X\left(e^{j(\omega-2 \pi m / M)}\right)
$$

where $V_{0}\left(e^{j \omega}\right)$ is the distortion function and $V_{m}\left(e^{j \omega}\right), m=$ $1,2, \ldots, M-1$ are the aliasing functions with

$$
V_{m}\left(e^{j \omega}\right)=\frac{1}{M} \sum_{k=1}^{K} B_{k}\left(e^{j(\omega-2 \pi m / M)}\right) C_{k}\left(e^{j \omega}\right)
$$

for $m=0,1, \ldots, M-1$. As can be seen from (8), for PR, $V_{0}\left(e^{j \omega}\right)=1$ and $V_{m}\left(e^{j \omega}\right)=0, m=1,2, \ldots, M-1$. Now, assume that for $L$ different combinations of the $K$ user band locations, the sampling instants $m_{k}, k=1,2, \ldots, K$ are selected such that $\mathbf{A}$ in (5) is an invertible matrix. In the proposed method, since the subfilters, $F_{k}(z)$ and $G_{k}(z)$, in the analysis FB are fixed based on the sampling instants, whenever a new combination of the $K$ user band locations is selected, it is only required to redetermine the complex constants $\mathbf{c}_{k}$. As can be seen from (5), the new $\mathbf{c}_{k}$ can be determined using matrix inversion.

The coefficients of the fixed subfilters $F_{k}(z), G_{k}(z)$, and $C_{k}(z)$ are determined offline such that, for each required combination of the $K$ user band location, $V_{0}\left(e^{j \omega}\right)$ and $V_{m}\left(e^{j \omega}\right)$, $m=1,2, \ldots, M-1$, approximate unity and zero, respectively, according to

$$
\begin{gathered}
\left|V_{0}\left(e^{j \omega}\right)-1\right| \leq \delta_{0} \quad \omega \in \Omega_{r 0}, \\
\left|V_{m}\left(e^{j \omega}\right)\right| \leq \delta_{1} \quad \omega \in \Omega_{r m}
\end{gathered}
$$

for $m=1,2, \ldots, M-1$. Here, $\delta_{0}$ and $\delta_{1}$ are the maximum approximation errors for the distortion function and aliasing functions, respectively, while $\Omega_{r 0}, r=1,2, \ldots, L$, are the active subband locations and $\Omega_{r m}, m=1, \ldots, M-1$, represent the shifted versions of the active subbands that fall into the band $[0, \pi]$.

\section{DESIGN EXAMPLE}

In this section, we illustrate the proposed reconfigurable reconstruction scheme with the help of a design example. In this example, it is assumed that, the total bandwidth is divided into $M=16$ granular bands. It is further assumed that there are three active users and $K=4$ active bands. At any given time frame, one user occupies two overlapping active granularity bands while each of the other two users occupy only one active granularity band. Also, we assume that, at any given time frame, the users can be allocated either the granularity bands $\{[3,4], 7,11\}$ or the bands $\{3,7,[11,12]\}$. For the above two possible combination of band locations, we used the sub-Nyquist sampling points, $m=0,4,7,13$, which ensures that $\mathbf{A}$ in (5) is an invertible matrix. In order to have practical filters, a transition band was included in each user band. Hence, for the two different combination of band locations, the information containing frequency bands were assumed to be $\{[3.2,4.8],[7.2,7.8],[11.2,11.8]\} \times \pi / 16$ and $\{[3.2,3.8],[7.2,7.8],[11.2,12.8]\} \times \pi / 16$, respectively. The reconstructor was designed such that, after reconstruction, the aliasing terms are below $-40 \mathrm{~dB}$. The prototype filter for the fixed synthesis FB was designed ${ }^{2}$ to be a power-symmetric lowpass filter of order 262 and with cutoff frequency at $0.6 \pi / 32$. It was found that, for the eight subfilters, $F_{k}(z)$ and $G_{k}(z)$, a filter order of 14 was sufficient to keep the aliasing terms below $-40 \mathrm{~dB}$. Thus, for the proposed method, the overall order of the reconstructor is 486 but with a computational complexity of around 30 (the exact value depends on how the $M \times 2 M$ transform block is implemented) multiplications per output sample due to the efficient realization in Fig. 2. At the same time, using the corresponding straightforward implementation in [2] would require a reconstructor with four synthesis filters of order around 150 and 37 multiplications per output sample. Also, during reconfiguration, only 32 multipliers need to be updated in the proposed method while in [2] around 600 multipliers have to be updated.

Table I shows the values corresponding to the complex constants $c_{k m_{l}}$ obtained for the two different combinations of user band locations. Figure 3 shows all the distortion and aliasing terms of the reconstructor for the two possible combinations of user band locations. It can be seen that, in the required bands, the aliasing terms are not greater than $-40 \mathrm{~dB}$ which validates the reconfigurability between the two different combinations of user band locations. The reconfigurability of the reconstructor is illustrated, in Fig. 4 and Fig. 5, by configuring it for one set of active band locations and using it to reconstruct a subNyquist sampled multi-tone input with tones in the active band region. The spectrum without reconstruction, in Figs. 4 and 5, corresponds to the spectrum of the sub-Nyquist sampled signal with zeros inserted into the time instants where the samples

\footnotetext{
${ }^{2}$ In this short paper, the prototype filter for $C_{k}(z)$ is designed and fixed before determining the coefficients of the subfilters $F_{k}(z)$ and $G_{k}(z)$.
} 
TABLE I

VALUES OF THE COMPLEX CONSTANT $c_{k m_{\ell}}$ FOR THE TWO DIFFERENT COMBINATION OF BAND LOCATIONS.

\begin{tabular}{|c|c|c|c|c|}
\hline \multicolumn{5}{|c|}{ User band locations: $\{3,7,[11,12]\}$} \\
\hline$m$ & 0 & 4 & 7 & 13 \\
\hline$n_{\ell}=3$ & $0.2500+j 0.3536$ & $-0.2500+j 0.3536$ & 0.3536 & -0.3536 \\
\hline$n_{\ell}=7$ & 0.5 & 0.5 & 0 & 0 \\
\hline$n_{\ell}=11$ & $-j 0.3536$ & $-j 0.3536$ & $-0.3536+j 0.3536$ & $0.3536+j 0.3536$ \\
\hline$n_{\ell}=12$ & 0.25 & -0.25 & $-j 0.3536$ & $-j 0.3536$ \\
\hline \hline \multicolumn{5}{|c|}{ User band locations: $\{[3,4], 7,11\}$} \\
\hline$m$ & 0 & 4 & 7 & 13 \\
\hline$n_{\ell}=3$ & $j 0.3536$ & $j 0.3536$ & $0.3536+j 0.3536$ & $-0.3536+j 0.3536$ \\
\hline$n_{\ell}=4$ & 0.25 & -0.25 & $-j 0.3536$ & $-j 0.3536$ \\
\hline$n_{\ell}=7$ & 0.5 & 0.5 & 0 & 0 \\
\hline$n_{\ell}=11$ & $0.2500-j 0.3536$ & $-0.2500-j 0.3536$ & -0.3536 & 0.3536 \\
\hline
\end{tabular}
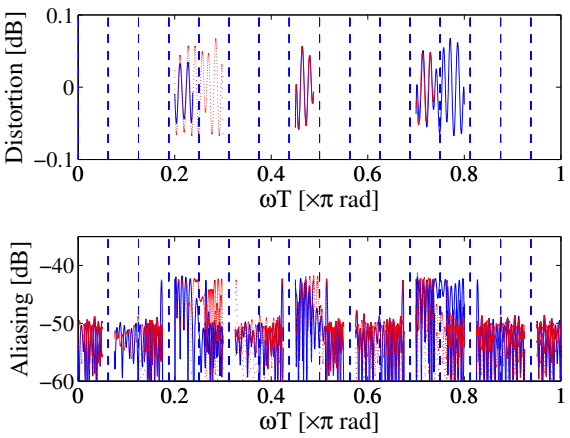

Fig. 3. Plot of the distortion function $V_{0}\left(e^{j \omega)}\right)$ and aliasing functions $V_{m}\left(e^{j \omega)}\right), m=1,2, \ldots, M-1$, for the active band combinations, $\{[3,4], 7,11\}$ (red-dotted) and $\{[3,7,[11,12]\}$ (blue-continuous), in the design example.

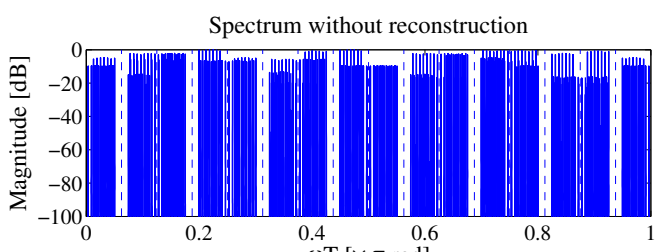

Spectrum after reconstruction

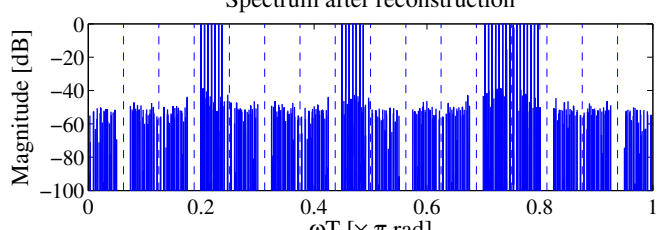

Fig. 4. Reconstruction of sub-Nyquist sampled multi-tone signals with tones in the three user bands, $\{[3,7,[11,12]\}$, after passing through the reconstructor in the design example.

are missing.

\section{Conclusion}

This paper introduced a reconfigurable reconstruction scheme for sub-Nyquist sampled sparse multi-band signals. By describing the reconstruction in terms of both the analysis and synthesis FB, substantial reduction in the design and implementation complexity of the reconstructor is achieved as shown in an example. Since the polyphase components of

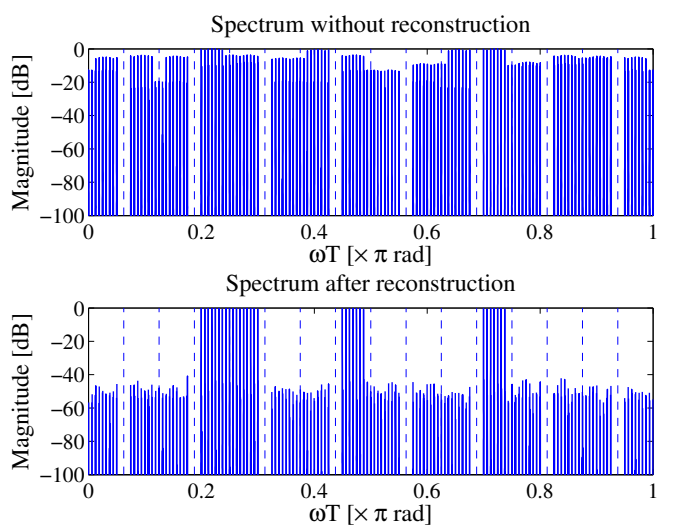

Fig. 5. Reconstruction of sub-Nyquist sampled multi-tone signals with tones in the three user bands, $\{[3,4], 7,11\}$, after passing through the reconstructor in the design example.

the filters in the analysis FB are generalized FD filters, all the analysis filters are expressed using a common set of fixed subfilters which are designed offline. The different analysis filters are then realized using different sets of multipliers. When the reconstructor is reconfigured online, only the coefficients of these multipliers have to be redetermined thus lowering the complexity of the online redesign block.

\section{REFERENCES}

[1] M. Mishali and Y. Eldar, "Sub-Nyquist sampling," IEEE Signal Processing Magazine, vol. 28, no. 6, pp. 98-124, Nov 2011.

[2] Y.-P. Lin and P. Vaidyanathan, "Periodically nonuniform sampling of bandpass signals," IEEE Transactions on Circuits and Systems II: Analog and Digital Signal Processing, vol. 45, no. 3, pp. 340-351, Mar 1998.

[3] W. C. Black and D. A. Hodges, "Time interleaved converter arrays," IEEE J. Solid-State Circuits, vol. 15, no. 6, pp. 1022-1029, Dec. 1980.

[4] A. Owrang, M. Viberg, M. Nosratinia, and M. Rashidi, "A new method to compute optimal periodic sampling patterns," in Proc. IEEE Digital Signal Processing and Signal Processing Education Workshop, Sedona, Arizona, USA, Jan. 4-7 2011, pp. 259-264.

[5] H. Johansson and A. Eghbali, "FIR filter with variable fractional delay and phase shift: Efficient realization and design using reweighted $\ell_{1}$-norm minimization," in Proc. IEEE Int. Symp. Circuits Syst., Beijing, China, May 19-23 2013.

[6] P. P. Vaidyanathan, Multirate Systems and Filter Banks. Prentice-Hall, Englewood Cliffs, NJ, USA, 1993.

[7] A. K. M. Pillai and H. Johansson, "Efficient recovery of sub-Nyquist sampled sparse multi-band signals using analysis and modulated synthesis filter banks," in preparation. 